\title{
On Computing Cluster Centers of Trapezoidal Fuzzy Numbers
}

\section{S. Sreenivasan, B. J. Balamurugan}

\begin{abstract}
In this paper we compute cluster centers of trapezoidal fuzzy numbers through fuzzy c means clustering algorithm and kernel based fuzzy c means clustering algorithm. A new complete metric distance between the trapezoidal fuzzy numbers is used to compute the cluster centers on the set of trapezoidal fuzzy numbers.
\end{abstract}

Keywords: Fuzzy clustering, Kernel function, Trapezoidal fuzzy numbers, Fuzzy C number clustering algorithms.

\section{INTRODUCTION}

In the history of fuzzy clustering, Dunn [3] fuzzy c means (FCM) clustering method proposed by and Bezdek [1] improved the algorithm, Dubois, D and Prade, H.[2]. Based on the similar idea of FCM construction, Yang and Ko [11] proposed new type of fuzzy clustering procedure called fuzzy c numbers (FCN) algorithm. However, Hung. W.L [6] explained the drawbacks of FCN over FCM and provided the alternate fuzzy c numbers algorithm (AFCN). Based on the concept of robust statistics, the AFCN algorithm improves on the weakness found in FCN. In [10] we have computed cluster centers of trapezoidal fuzzy numbers using two different metric distances.

In this paper, we use a new metric distance $d_{f}$ to discuss the robustness of the fuzzy c means type algorithms.

The section II describes the definition of trapezoidal fuzzy number and the distance $d_{f}$, introduced by Hadi Sadoghi et.al [4], between two trapezoidal intervals. Two clustering algorithms $[10,13,14]$ on the space of all trapezoidal fuzzy intervals are described in the section III based on the distance $d_{f}$. Numerical example is given to compare the effectiveness of the two algorithms FCM and KFCM in section IV. Conclusions are given in section V.

\section{PRELIMINARIES}

Definition 1: $X=\left(m_{1}, m_{2}, \alpha, \beta\right)_{L R}$ with $\alpha>0, \beta>0$ is called a LR-type fuzzy number if

Revised Manuscript Received on December 20, 2019.

* Correspondence Author

S. Sreenivasan*, School of Advanced Sciences, VIT University, Chennai Campus, Chennai, India. Email: sreenivasan.s2017@vitstudent.ac.in

B. J. Balamurugan, School of Advanced Sciences, VIT University, Chennai Campus, Chennai, India. Email: balamurugan.bj@vit.ac.in

$$
X(x)=\left\{\begin{array}{lr}
L\left(\frac{m_{1}-x}{\alpha}\right), & \text { for } x \leq m_{1} \\
1, & \text { for } m_{1} \leq x \leq m_{2} \\
L\left(\frac{x-m_{2}}{\beta}\right), & \text { for } x \geq m_{2}
\end{array}\right.
$$

We note that the trapezoidal type of fuzzy number is the simplest and most used one among $L R$-type fuzzy intervals.

Let $X=\left(m_{1}, m_{2}, \alpha, \beta\right)_{L R}$ be a $L R$-type fuzzy interval. If $L$ and $R$ of the form

$$
T(x)=\left\{\begin{array}{lr}
1-x, & 0 \leq x \leq 1 \\
0, & \text { Otherwise }
\end{array}\right.
$$

Then

$$
X(x)=\left\{\begin{array}{lr}
1-\frac{m_{1}-x}{\alpha}, & \text { for } x \leq m_{1} \\
1, & \text { for } m_{1} \leq x \leq m_{2} \\
1-\frac{x-m_{2}}{\beta}, & \text { for } x \geq m_{2}
\end{array}\right.
$$

is said to be a trapezoidal fuzzy interval

Let the set $F_{L R}(R)$ of all $L R$-type fuzzy intervals. Hadi Sadoghi et.al [4] gives a metric distance $d_{f}^{2}(X, Y)$ based on $\quad$ Yang[11] between $X=\left(m_{1 x}, m_{2 x}, \alpha_{x}, \beta_{x}\right)_{L R}$ and $Y=\left(m_{1 y}, m_{2 y}, \alpha_{y}, \beta_{y}\right)_{L R}$ which are in $F_{L R}(R)$ as follows:

$d_{f}^{2}(X, Y)=\frac{1}{4}\left(g_{-}^{2}+g_{+}^{2}+\left(g_{-}-\left(\alpha_{x}-\alpha_{y}\right)\right)^{2}+\left(g_{+}-\left(\beta_{x}-\beta_{y}\right)\right)^{2}\right)$

Where $\quad g_{-}=2\left(m_{1 x}-m_{1 y}\right)-\left(m_{2 x}-m_{2 y}\right) \quad$ and $g_{-}=2\left(m_{1 x}-m_{1 y}\right)+\left(m_{2 x}-m_{2 y}\right)$

Theorem 1: $\left(F_{L R}(R), d_{f}\right)$ is metric.

Theorem 2: $\left(F_{L R}(R), d_{f}\right)$ is complete.

\section{FUZZY CLUSTERING ALGORITHMS}

In this section we recall FCM clustering algorithm[1] and KFCM clustering algorithm[12,13].

Let the set $X=\left\{\tilde{X}_{1}, \tilde{X}_{2}, \tilde{X}_{3}, \ldots, \tilde{X}_{n}\right\}$ of fuzzy numbers in $F_{L R}(R)$ with $\tilde{X}_{k}=\left(m_{1 x_{k}}, m_{2 x_{k}}, \alpha_{x_{k}}, \beta_{x_{k}}\right), 1 \leq k \leq n$. Let $c$ be the number of clusters.

Let $V=\left\{\tilde{V}_{i} \mid 1<i \leq c\right\}$ be 
the $\tilde{V}_{i}=\left(m_{1 v_{i}}, m_{2 v_{i}}, \alpha_{v_{i}}, \beta_{v_{i}}\right)$ and

centers,

where between $\tilde{X}_{k}$ and $\tilde{V}_{i}$.

\section{A. Fuzzy c Means Clustering}

The set $X$ can be divided into c fuzzy subsets using FCM by minimizing the objective function

$$
J_{F C M}(U, V)=\sum_{i=1}^{c} \sum_{k=1}^{n} u_{i k}^{m} d_{i k}^{2}
$$

where $d_{i k}=d\left(\tilde{V}_{i}, \tilde{X}_{k}\right)$ and $u_{i k}$ is the membership value of the fuzzy number $\tilde{X}_{k}$ in cluster $i$ with $\sum_{i=1}^{c} u_{i k}=1$, the fuzziness index $m \in[1, \infty)$ and $U=\left(u_{i k}\right)_{n \times c}$ is a fuzzy c partition matrix.

The Parameters of FCM are calculated by improving the function $\min J$ step by step according to the formulas below:

$$
\begin{aligned}
& m_{1_{i}}=\frac{\sum_{k=1}^{n} u_{i k}^{m} m_{1 x_{k}}}{\sum_{k=1}^{n} u_{i k}^{m}} \\
& m_{2 v_{i}}=\frac{\sum_{k=1}^{n} u_{i k}^{m} m_{2 x_{k}}}{\sum_{k=1}^{n} u_{i k}^{m}} \\
& \alpha_{v_{i}}=\frac{\sum_{k=1}^{n} u_{i k}^{m} \alpha_{x_{k}}}{\sum_{k=1}^{n} u_{i k}^{m}} \\
& \beta_{v_{i}}=\frac{\sum_{k=1}^{n} u_{i k}^{m} \beta_{x_{k}}}{\sum_{i k}^{n} u_{i k}^{m}} \\
& \sum_{j=1}^{c} d_{j k}^{\frac{-2}{m-1}}
\end{aligned}
$$

Based on these formulas, on trapezoidal fuzzy numbers we use the following fuzzy c means clustering algorithm.

Step1: Let the fuzziness index be $m>1$, let the number of partitions $c=\{2,3,4, \ldots,(n-1)\}$ and let any $\varepsilon>0$. Choose $U^{(0)}$ be the fuzzy c partition matrix initially and take $t=0$

Step2:

Calculate cluster centers $\quad V^{(t)}=\left\{\tilde{V}_{i}^{(t)} \mid 1<i \leq c\right\} \quad$ using $\quad U^{(t)} \quad$ and equations $(1),(2),(3)$ and $(4)$.

Step3: Improve $U^{(t)}$ as $U^{(t+1)}$ using $V^{(t)}$ and equation(5).

Step4: Compute $E^{k}=\operatorname{Max}_{i, k}\left\{\left|u_{i k}^{(t+1)}-u_{i k}^{(t)}\right|\right\}$, if $E^{k} \leq \varepsilon$, stop. Otherwise set $U^{(t+1)}=U^{(t)}$ and move to step 2 .

\section{B. Kernel Fuzzy c Means Clustering}

A kernel function $[5,7,8,9]$ maps nonlinearly the input data space into high dimensional feature space $H$. In the M-dimensional space $R^{M}$, let the unlabeled set $X=\left\{\tilde{X}_{1}, \tilde{X}_{2}, \tilde{X}_{3}, \ldots, \tilde{X}_{n}\right\}$ and let

$\Phi: R^{M} \rightarrow H, \tilde{X} \rightarrow \Phi(\tilde{X})$

To find the dot product in the high dimensional feature space, we can use the kernel function $K\left(\tilde{X}_{i}, \tilde{X}_{j}\right)=\Phi\left(\tilde{X}_{i}\right) \cdot \Phi\left(\tilde{X}_{j}\right)$

Examples of kernel function

- Polynomial:

$$
K\left(\tilde{X}_{i}, \tilde{X}_{j}\right)=\left(\gamma \tilde{X}_{i}^{T} \cdot \tilde{X}_{j}+c\right)^{d}, \gamma>0, d \in N
$$

- Sigmoid: $K\left(\tilde{X}_{i}, \tilde{X}_{j}\right)=\tanh \left(\gamma \tilde{X}_{i}^{T} \cdot \tilde{X}_{j}+c\right)^{d}, \gamma>0$

- RBF: $K\left(\tilde{X}_{i}, \tilde{X}_{j}\right)=\exp \left(-\gamma\left\|\tilde{X}_{i}-\tilde{X}_{j}\right\|^{2}\right), \gamma>0$

where $\quad \gamma, c, d \quad$ are kernel parameters.

Since,

$$
\begin{aligned}
\left\|\Phi\left(\tilde{X}_{k}\right)-\Phi\left(\tilde{V}_{i}\right)\right\|^{2}= & \left(\Phi\left(\tilde{X}_{k}\right)-\Phi\left(\tilde{V}_{i}\right)\right)^{T}\left(\Phi\left(\tilde{X}_{k}\right)-\Phi\left(\tilde{V}_{i}\right)\right) \\
= & \Phi\left(\tilde{X}_{k}\right)^{T} \Phi\left(\tilde{X}_{k}\right)-\Phi\left(\tilde{X}_{k}\right)^{T} \Phi\left(\tilde{V}_{i}\right) \\
& \quad-\Phi\left(\tilde{V}_{i}\right)^{T} \Phi\left(\tilde{X}_{k}\right)+\Phi\left(\tilde{V}_{i}\right)^{T} \Phi\left(\tilde{V}_{i}\right) \\
= & K\left(\tilde{X}_{k}, \tilde{X}_{k}\right)+K\left(\tilde{V}_{i}, \tilde{V}_{i}\right)-2 K\left(\tilde{X}_{k}, \tilde{V}_{i}\right)
\end{aligned}
$$

when the kernel function is chosen as RBF, $K\left(\tilde{X}_{k}, \tilde{X}_{k}\right)=1, K\left(\tilde{V}_{i}, \tilde{V}_{i}\right)=1$, then

$\left\|\Phi\left(\tilde{X}_{k}\right)-\Phi\left(\tilde{V}_{i}\right)\right\|^{2}=2\left(1-K\left(\tilde{X}_{k}, \tilde{V}_{i}\right)\right)$

The set $X$ can be divided into c fuzzy subsets using KFCM by minimizing the objective function

$$
J_{K F C M}(U, V)=\sum_{i=1}^{c} \sum_{k=1}^{n} u_{i k}^{m}\left(1-K\left(\tilde{X}_{k}, \tilde{V}_{i}\right)\right)
$$

The Parameters of kernel fuzzy c means are calculated by improving the function $\min J$ step by step according to the formulas below:

$$
m_{1 v_{i}}=\frac{\sum_{k=1}^{n} u_{i k}^{m} K\left(\tilde{X}_{k}, \tilde{V}_{i}\right) m_{1 x_{k}}}{\sum_{k=1}^{n} u_{i k}^{m}}
$$




$$
\begin{aligned}
& m_{2 v_{i}}=\frac{\sum_{k=1}^{n} u_{i k}^{m} K\left(\tilde{X}_{k}, \tilde{V}_{i}\right) m_{2 x_{k}}}{\sum_{k=1}^{n} u_{i k}^{m}} \\
& \alpha_{v_{i}}=\frac{\sum_{k=1}^{n} u_{i k}^{m} K\left(\tilde{X}_{k}, \tilde{V}_{i}\right) \alpha_{x_{k}}}{\sum_{k=1}^{n} u_{i k}^{m}} \\
& \beta_{v_{i}}=\frac{\sum_{k=1}^{n} u_{i k}^{m} K\left(\tilde{X}_{k}, \tilde{V}_{i}\right) \beta_{x_{k}}}{\sum_{k=1}^{n} u_{i k}^{m}} \\
& u_{i k}=\frac{\left[1-K\left(\tilde{X}_{k}, \tilde{V}_{i}\right)\right]^{\frac{-1}{m-1}}}{\sum_{j=1}^{c}\left[1-K\left(\tilde{X}_{k}, \tilde{V}_{j}\right)\right]^{\frac{-1}{m-1}}}
\end{aligned}
$$

Based on these formulas, on trapezoidal fuzzy numbers we use the following kernel fuzzy means clustering algorithm.

Step1: Let the fuzziness index be $m>1$, let the number of partitions $c=\{2,3,4, \ldots,(n-1)\}$ and let any $\varepsilon>0$. Choose $U^{(0)}$ be the fuzzy c partition matrix initially and take $t=0$.

Step2: Calculate

centers $\quad V^{(t)}=\left\{\tilde{V}_{i}^{(t} \mid 1<i \leq c\right\} \quad$ using $\quad U^{(t)} \quad$ and equations (6), (7), (8) and (9).

Step3: Improve $U^{(t)}$ as $U^{(t+1)}$ using $V^{(t)}$ and equation(10).

Step4: Compute $E^{k}=\operatorname{Max}_{i, k}\left\{\left|u_{i k}^{(t+1)}-u_{i k}^{(t)}\right|\right\}$, if $E^{k} \leq \varepsilon$, stop. Otherwise set $U^{(t+1)}=U^{(t)}$ and move to step 2 .

In the experiment, we used RBF kernel is used with the parameter $\gamma$ defined by

$$
\gamma=\left(\frac{\sum_{k=1}^{n} d\left(\tilde{X}_{k}, \bar{W}\right)}{n}\right)^{-1}
$$

with $\bar{W}=\left(\bar{m}_{1 w}, \bar{m}_{2 w}, \bar{\alpha}_{w}, \bar{\beta}_{w}\right)$ is the arithmetic mean, where

$$
\begin{gathered}
\bar{m}_{1 w}=\frac{\sum_{k=1}^{n} m_{1 x_{k}}}{n} \\
\bar{m}_{2 w}=\frac{\sum_{k=1}^{n} m_{2 x_{k}}}{n}
\end{gathered}
$$

$$
\begin{gathered}
\bar{\alpha}_{w}=\frac{\sum_{k=1}^{n} \alpha_{x_{k}}}{n} \\
\bar{\beta}_{w}=\frac{\sum_{k=1}^{n} \beta_{x_{k}}}{n}
\end{gathered}
$$

\section{NUMERICAL EXAMPLES}

In this section, we implement the algorithms with $m=2$ and $\varepsilon=0.001$. Consider the data set $G_{1}$ from Yang, M.S and Ko, C.H.[11] consisting of 30 trapezoidal fuzzy numbers given in "Fig. 1".

We run both algorithms on the data set $G_{1}$ with the complete metric distances $d_{f}$ and $c=3$. The corresponding

\begin{tabular}{|c|c|}
\hline & $\left(m_{1 v}, m_{2 v}, \alpha_{v}, \beta_{v}\right)$ \\
\hline & With distance $d_{f}^{2}(X, Y)$ \\
\hline & $V_{1} \quad(39.516,41.438,1.283,0.870)$ \\
\hline Algorithm-1 & $V_{2} \quad(24.077,26.081,0.909,1.043)$ \\
\hline & $(12.858,14.652,1.130,1.202)$ \\
\hline & $V_{1} \quad(39.021,40.917,1.228,0.841)$ \\
\hline Algorithm-2 & $V_{2} \quad(24.047,25.996,0.814,1.063)$ \\
\hline & $V_{3} \quad(14.133,15.795,1.146,1.166)$ \\
\hline
\end{tabular}
results are shown in "Table. I" and the cluster centers are shown in "Fig. 3".

\begin{tabular}{|c|c|}
\hline & $\left(m_{1 v}, m_{2 v}, \alpha_{v}, \beta_{v}\right)$ \\
\hline & With distance $d_{f}^{2}(X, Y)$ \\
\hline \multirow{3}{*}{ Algorithm-1 } & $V_{1} \quad(98.900,100.101,0.672,1.024)$ \\
\hline & $V_{2} \quad(37.561,39.540,1.283,0.867)$ \\
\hline & $(16.555,18.413,1.095,1.180)$ \\
\hline \multirow{3}{*}{ Algorithm-2 } & $V_{1} \quad(39.359,41.267,1.357,0.860)$ \\
\hline & $(24.138,26.116,0.865,1.050)$ \\
\hline & $(13.626,15.359,1.134,1.187)$ \\
\hline
\end{tabular}

Consider the set $G_{2}$ consisting $G_{1}$ and one more point $(98.020,100.000,1.630,0.710)$ called outlier point shown in "Fig. 2". Now on the set $G_{2}$ with the number of clusters $c=2$, we run both algorithms. The corresponding results are shown in "Fig. 4" and "Table. II".

Table- I: Cluster centers in set $G_{1}$

Table- II: Cluster centers in set $G_{2}$ 


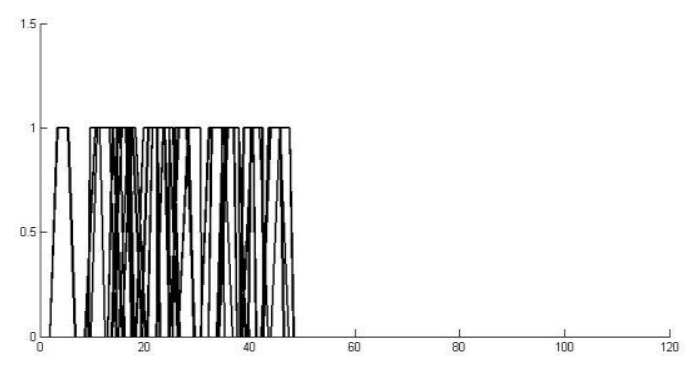

Fig. 1. Set $G_{1}$

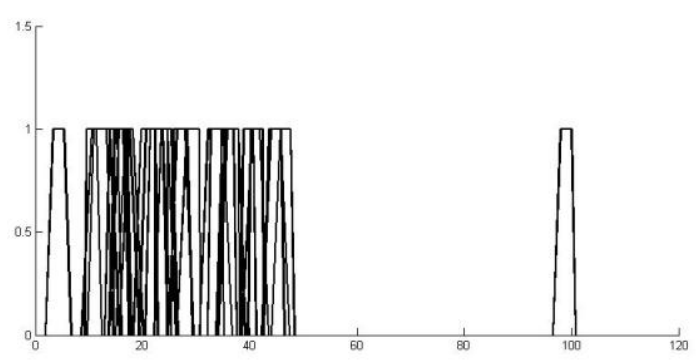

Set $G_{2}$

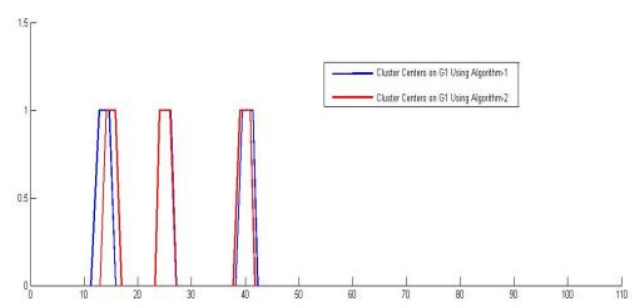

Cluster centers in set $G_{1}$

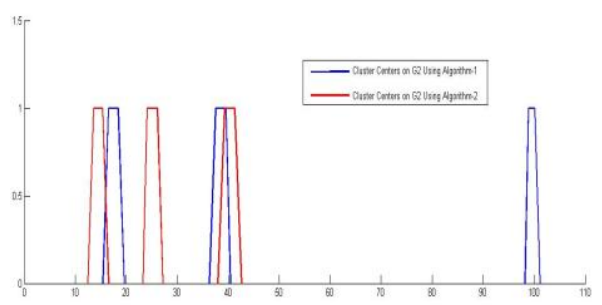

Cluster centers in set $G_{2}$

When we run Algorithm-1 and Algorithm-2 on data set $G_{1}$ with the distance $d_{f}$, the cluster centers are almost same. While running Algorithm-1 on the data set $G_{2}$ (With outlier point) gives poor result, but the Algorithm- 2 on $G_{2}$ provides almost the same result of $G_{1}$. That is the centers obtained by algorithm-1 on $G_{2}$ are away from the clusters whereas the centers obtained by Algorithm-2 on $G_{2}$ are within the cluster and coincides with the centers of $G_{1}$ data.

\section{CONCLUSION}

We used a different metric distance $d_{f}$ to calculate centers of clusters through the FCM clustering algorithm and kernel based KFCM algorithm on trapezoidal fuzzy numbers. We have written MATLAB programming to compute the centers. The results show that the KFCM clustering algorithm performs better than the FCM clustering algorithm.

\section{REFERENCES}

1. J. C. Bezdek, Pattern Recognition with Fuzzy Objective Function Algorithms, (Plenum Press, New York, 1981).

2. D Dubois and $\mathrm{H}$ Prade. Fuzzy sets and systems: Theory and applications, (Academic Press, New York, 1980).

3. J. C. Dunn, "A fuzzy relative of the ISODATA process and Its Use in Detecting Compact", well-Separated Cluster, J. Cybernet, 1974, 3, pp. $32-57$.

4. Hadi Sadoghi Yazdi, Mehri Sadoghi Yazdi, Abedin Vahedian, "Fuzzy Bayesian Classification of LR Fuzzy Numbers", IACSIT International Journal of Computer Theory and Engineering, Vol. 1, No. 5, December 2009.

5. R Herbrich, Learning Kernel Classifier, (MIT Press, Cambridge, MA 2002).

6. W. L. Hung and M. S. Yang, "Fuzzy Clustering on LR-Type fuzzy numbers With an Application in Taiwanese Tea Evaluation", Fuzzy sets and systems, 2005, 150(3), pp. 561 - 577.

7. Meena Tushir, Smriti Srivastava, "A New Kernel based Hybrid c Means Clustering model", IEEE international Fuzzy systems Conference, 2007.

8. K R Muller, S Mika, G Ratsch, K Tsuda, B Scholkopf, “An introduction to kernel-based learning algorithms", IEEE Transaction on Neural Networks 12(2), 2001, pp. 181-201.

9. Rong Lan, Jiu-Iun Fan, “A Fuzzy C Means Type Clustring Algorithm on triangular Fuzzy Numbers", Sixth international conference on Fuzzy Systems and Knowledge Discovery, 2009.

10. S Sreenivasan and B J Balamurugan, "Computing Cluster Centers of Trapezoidal Fuzzy Numbers Through Fuzzy C Means and Kernel Based Fuzzy C Means Clustering Algorithms with Two Metric Distances Using Matlab", International Journal of Civil Engineering and Technology (IJCIET) 9(10), 2018, pp. 1322-1330.

11. M S Yang, C H Ko, "On a Class of fuzzy c Numbers Clustering Procedure for Fuzzy Data”, Fuzzy sets and systems, 1996, 84(1), 49 - 60.

12. D Zhang, S Chen, "Fuzzy clustering kernel method", in: Proc of the Internat. Conf. on Control and Automation, 2002, pp. 123-127.

13. S Zhou, J Gan, "Mercer kernel fuzzy c means algorithm and prototypes of clusters", in: Proc. of Conf. on Internat. Data Engineering and Automated Learning, Vol 3177, 2004, pp. 613-618.

\section{AUTHORS PROFILE}

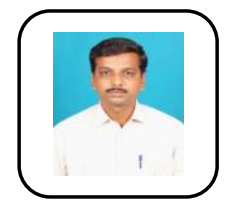

S. Sreenivasan, received his M.Sc. and M.Phil degrees in Mathematics from the University of Madras, Chennai, India. He has more than 20 years of teaching experience at Undergraduate and Postgraduate level courses. He is currently pursuing Ph.D. in Fuzzy Graph. He has published 5 research papers in various journals and conference proceedings. His research interest includes graph theory, Fuzzy Clustering and fuzzy logic.

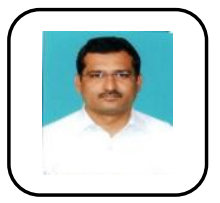

Dr. B. J. Balamurugan, received his $\mathrm{Ph} . \mathrm{D}$ degree in Mathematics from the University of Madras, Chennai, India. Currently, he is an Assistant Professor (Senior) of Mathematics in the School of Advanced Sciences at VIT University, Chennai Campus, Chennai, India. He has more than 22 years of teaching experience at Undergraduate and Postgraduate level courses. Dr. B. J. Balamurugan has published more than 30 research papers in various journals and conference proceedings. His research interest includes graph theory, graph grammars, fuzzy logic and Petri nets. 